\title{
Optimizing pharmacotherapy in elderly patients: the role of pharmacists
}

This article was published in the following Dove Press journal:

Integrated Pharmacy Research and Practice

II August 2015

Number of times this article has been viewed

Jeannie K Lee'

Samah Alshehri ${ }^{1,2}$

Hussam I Kutbi ${ }^{1,2}$

Jennifer R Martin ${ }^{1,3}$

'Pharmacy Practice and Science, University of Arizona College of Pharmacy, Tucson, AZ, USA; 2Department of Clinical Pharmacy, King Abdulaziz University College of Pharmacy, Jeddah, Saudi Arabia; ${ }^{3}$ Arizona Health Sciences Library, University of Arizona, Tucson, AZ, USA

Correspondence: Jeannie K Lee

Pharmacy Practice and Science, University of Arizona College of Pharmacy, 1295

North Martin Avenue, Tucson, AZ

85721-0202, USA

$\mathrm{Tel}+\mathrm{I} 5206269419$

$\mathrm{Fax}+\mathrm{I} 5206267355$

Email jlee@pharmacy.arizona.edu
Abstract: As the world's population ages, global health care systems will face the burden of chronic diseases and polypharmacy use among older adults. The traditional tasks of medication dispensing and provision of basic education by pharmacists have evolved to active engagement in direct patient care and collaborative team-based care. The care of older patients is an especially fitting mission for pharmacists, since the key to geriatric care often lies with management of chronic diseases and polypharmacy use, and preventing harmful consequences of both. Because most chronic conditions are treated with medications, pharmacists, with their extensive training in pharmacotherapy and pharmacokinetics, are in a unique and critical position in the management of them. Pharmacists have the expertise to detect, resolve, and prevent medication errors and drug-related problems, such as overtreatment, undertreatment, adverse drug events, and nonadherence. Pharmacists are also competent in critically reviewing and applying clinical guidelines to the care of individual patients, and in some instances confront the lack of data (common in older adults) to provide the best possible patient-centered care. The current review aimed to depict the evidence of geriatric pharmacy care, demonstrate current impact of pharmacists' interventions on older patients, survey the tools used by pharmacists to provide effective care, and explore their role in pharmacotherapy optimization in elders. The findings of the current review strongly support previous studies that showed positive impact of pharmacists' interventions on older patients' health-related outcomes. There is a clear role for pharmacists working directly or collaboratively to improve medication use and management in older populations. Therefore, in global health care systems, teams caring for elders should involve pharmacists to optimize pharmacotherapy.

Keywords: pharmacist, elderly, older adult, pharmacotherapy, medication, polypharmacy

\section{Introduction}

As the world population ages, health systems globally will face the burden of chronic disease and polypharmacy management in older patients. Within efforts to identify the optimal model to care for older adults and to increase formation of effective interprofessional health care teams, pharmacists play important and distinct roles. Pharmacists are increasingly becoming involved in primary care-delivery models, such as patient-centered medical homes, where team-based care is provided in a patient-centered, comprehensive, coordinated and accessible manner with a focus on quality and safety. ${ }^{1,2}$ Pharmacists are also collaborating in interprofessional health care teams, ${ }^{3-5}$ providing consultation services, ${ }^{6,7}$ and leading patient care interventions focused on impactful outcomes. ${ }^{8}$ This movement of pharmacists' involvement in direct patient care away from the traditional medication-dispensing functions is widespread 
globally, including those countries with low- to middleincome status. ${ }^{9,10}$ Within the interprofessional health care teams, pharmacists are making a positive impact on various health outcomes among diverse patient populations, including older adults. ${ }^{3-5}$ Disease management targeting chronic conditions that are often treated with medications is commonly performed by pharmacists, such as caring for patients with cardiovascular disease risk factors, ${ }^{11}$ hypertension, ${ }^{12,13}$ dyslipidemia, ${ }^{14}$ diabetes, ${ }^{15-17}$ heart failure,${ }^{18}$ chronic obstructive pulmonary disease (COPD),${ }^{19}$ and insomnia. ${ }^{20}$ Studies evaluating chronic disease-management services provided by pharmacists frequently attempt to measure the impact on medication adherence, disease control using clinical end points, health service utilization (eg, emergency department visits, hospital admission), and health care-related costs. ${ }^{6,19,21,22}$ Pharmacists are also expanding their practice settings to broader health care settings and transition points, creating models of service that target vulnerable patients and gaps in the system to generate cost savings. ${ }^{6,8,23-25}$

Because older adults have multiple chronic conditions and often use polypharmacy, they are an ideal patient population for pharmacists' interventions. Polypharmacy occurs when patients are required to use multiple medications concurrently (four to nine, depending on the literature) for their health conditions. Multiple studies point to the dangers of polypharmacy use among older adults, including potentially inappropriate medications (PIMs), anticholinergic burden, drug-drug interaction-related adverse drug reactions. ${ }^{26-30}$ The prevalence of polypharmacy use and inappropriate prescribing in the elderly is a global phenomenon reported in multiple countries. ${ }^{4,31,32}$ The consequential hospitalization, other health care-service utilization, and harm to patients (eg, falls and fractures) are the measures used to demonstrate the damage to patients, health care systems, and cost structure. ${ }^{26,33}$

\section{Objectives and procedures}

Optimization of pharmacotherapy is central to pharmacist function in caring for and impacting elderly patients. This review explores: 1) the evidence of geriatric pharmacy care, 2 ) the current impact of pharmacist interventions on older patients, 3) tools that have been used by pharmacists, and 4) the pharmacist's role in pharmacotherapy optimization in elders.

To gather and assess the most current impact of pharmacists' interventions on older patients (objective 2), we conducted a brief review of the literature. For this review, four databases that most commonly cite quality studies on pharmacist work in clinical settings were searched:
PubMed/Medline, Embase, International Pharmaceutical Abstracts, and PsycInfo. Search terms incorporated both keywords and controlled vocabulary for the databases used. Example terms included pharmacist, pharmacy, pharmacy service, aged, frail elderly, outpatients, inpatients, direct patient care, medication management, medication adherence, polypharmacy, disease management, and drug therapy. The search was restricted to the English language, and included publications from the last 10 years to the date the searches were conducted (November 2014) to capture the most recent and widely viewed information. Publications excluded from the results were letters, editorials, and conference proceedings. Review articles, systematic reviews, and meta-analyses were separately kept to be reviewed and discussed under the evidence of geriatric pharmacy care, tools used by pharmacists, and role of pharmacists in geriatric care sections.

A preliminary review of study titles and abstracts was conducted to exclude articles that did not meet the inclusion criteria: 1) subjects aged 65 years and older, 2) described distinguishable pharmacist intervention, 3) utilized comparative design, and 4) measured patient-related health outcomes. A dual review was performed using abstracts and full text of 16 publications, and ten studies were included as showing current impact of pharmacists in the care of older adults. Data extraction was conducted by two reviewers independently, then checked by a third reviewer.

\section{Existing evidence of geriatric pharmacy care}

Several reviews have addressed the evaluation of pharmacistdelivered disease-management programs. A systematic review and meta-analysis assessed care provided by pharmacists in managing cardiovascular disease risk factors, and found a favorable impact of pharmacist care among 30 studies in reducing blood pressure, total cholesterol, low-density lipoprotein cholesterol, and smoking. ${ }^{11}$ Another systematic review and meta-analysis of pharmacist management of hypertension showed a sensitive association between pharmacists' interventions and reductions in systolic blood pressure. ${ }^{14}$ A literature review conducted by Ponniah et al assessed the postdischarge management of heart-failure patients by pharmacists. ${ }^{34}$ The review included seven studies with pharmacists using telephone calls or home-visit follow-up, outpatient heart-failure clinic visit, and homebased intervention. The pharmacist-delivered heart-failure disease-management program yielded positive outcomes for patients, such as fewer readmissions and out-of-hospital 
deaths, improved medication adherence and exercise capacity, reduced clinical events, and decreased hospitalizations. ${ }^{34}$ An additional systematic review looking at pharmacist care in directed or collaborative fashion to manage heart failure retrieved 12 randomized controlled trials (RCTs) that significantly lowered all-cause hospitalization and heart failure-related hospitalization, with collaborative care having greater effects in reducing heart failure-related hospitalization compared to directed care. ${ }^{18}$

In 2013, Lee et al published a systematic review and metaanalyses that outlined geriatric care by pharmacists in health care teams in the USA. ${ }^{4}$ This study aimed to identify comparative studies that evaluated direct patient interventions by pharmacists with published results cited in PubMed/Medline, Ovid/Medline, ABI/Inform, Health Business Fulltext Elite, Academic Search Complete, International Pharmaceutical Abstracts, PsycInfo, Cochrane Database, and ClinicalTrials. gov since the inception of the databases through July 2012. Included studies reported interventions by pharmacists to patients aged 65 years and older, had comparison design, and measured patient-based health outcomes. Among the 20 articles with adequate data to perform meta-analyses, there were varied study designs (RCTs to retrospective cohort), small $(n=36)$ to large $(n=4,218)$ studies, and mixed conditions being managed in diverse settings. ${ }^{4}$ Twelve of the 20 studies included in this meta-analyses were published within the past 10 years, the time frame for the current review. ${ }^{21,35-45}$ Although pharmacists' interventions varied, all but one study ${ }^{41}$ published within the past 10 years used a technical intervention, such as therapeutic change, anticoagulation management, and blister-pack medications, though five of those studies used it in combination with an educational intervention (patient or medication education). Meta-analyses' data, using forest plots of study outcomes, significantly favored pharmacist care versus comparison in all outcome categories reviewed: therapeutic, ${ }^{21,40,42,43}$ safety, ${ }^{35,37,39,42,45}$ hospitalization, ${ }^{36,38,39,41,44}$ and adherence ${ }^{21,40,43}(P<0.001){ }^{4}$ This review highlighted the importance of having pharmacist integration in interprofessional team care of older patients, and served as a call to reform of the payment system and provider status of pharmacists in the USA.

\section{Current impact of pharmacists in the care of older adults}

A total of 321 articles were located, and 12 duplicates were removed. After screening abstracts and full text of 16 possible studies, ten studies were included in the current review that reported pharmacists' impact and were published in the past
10 years, but had not been included in the previous systematic review and meta-analyses on geriatric pharmacy care in the USA. ${ }^{4}$ The literature search for the current review was done globally, and not limited to pharmacists' work in the USA. All included studies were published between 2007 and 2014, provided distinguishable interventions by pharmacists to patients aged 65 years and older, and measured clinical outcomes in comparison to non-pharmacist interventions or controls. ${ }^{46-55}$ Table 1 describes the characteristics of the studies, including study design with the Scottish Intercollegiate Guidelines Network rating ${ }^{56}$ for bias assessment, total number of subjects, patient age, percentage of male subjects, target disease or condition, outcomes measured, and primary results. There were four RCTs, ${ }^{50-52,54}$ three prospective cohort studies,,${ }^{46,47,55}$ one before-and-after study evaluating the same patient cohort before and after intervention), ${ }^{53}$ and two before-and-after studies evaluating different patient cohorts before and after intervention. ${ }^{48,49}$ The number of study subjects ranged from 25 in a before-and-after study ${ }^{48}$ to 59,680 in an RCT. ${ }^{52}$ The majority of included patients were female in seven of ten studies. Seven studies did not focus on one specific disease or condition, but targeted medication-specific outcomes, such as PIMs and the Medication Appropriateness Index (MAI) in the inpatient setting. ${ }^{46,49,51-55}$ One study each focused on patients having anticoagulation, ${ }^{47} \mathrm{COPD}$ exacerbation, ${ }^{48}$ and orthopedic, colorectal, or vascular surgery. ${ }^{50}$

Table 2 depicts study settings, interventions, and pharmacists' activities involved in the interventions performed in the included studies. Nine studies were conducted in a hospital, ${ }^{46-51,53-55}$ with four specifically in a geriatric care setting, ${ }^{49,53-55}$ while one study was conducted at an outpatient health maintenance organization. ${ }^{52}$ Pharmacist-delivered interventions included medication reconciliation; ${ }^{48,55}$ and medication-management interventions included systematic medication care plans, ${ }^{46}$ warfarin dosing, ${ }^{47}$ interim residential care medication administration charts, ${ }^{49}$ pharmacotherapy changes ${ }^{50} \mathrm{PIMs}$, potential prescription-omission resolution, ${ }^{51,52}$ and an in-hospital pharmaceutical care program. ${ }^{54}$ All studies involved pharmacists providing direct and/or health care team-based collaborative interventions to older patients.

As described in Table 1, outcome measures assessed varied in the included studies. Four studies used the MAI as an outcome measure, some among other measures, ${ }^{46,51,53,54}$ while one study tracked PIMs specifically. ${ }^{52}$ Clinical and safety outcomes were evaluated by other studies, ${ }^{47-50,55}$ with one study assessing an economic outcome (cost of readmission). ${ }^{48}$ Nine studies found positive impact by 


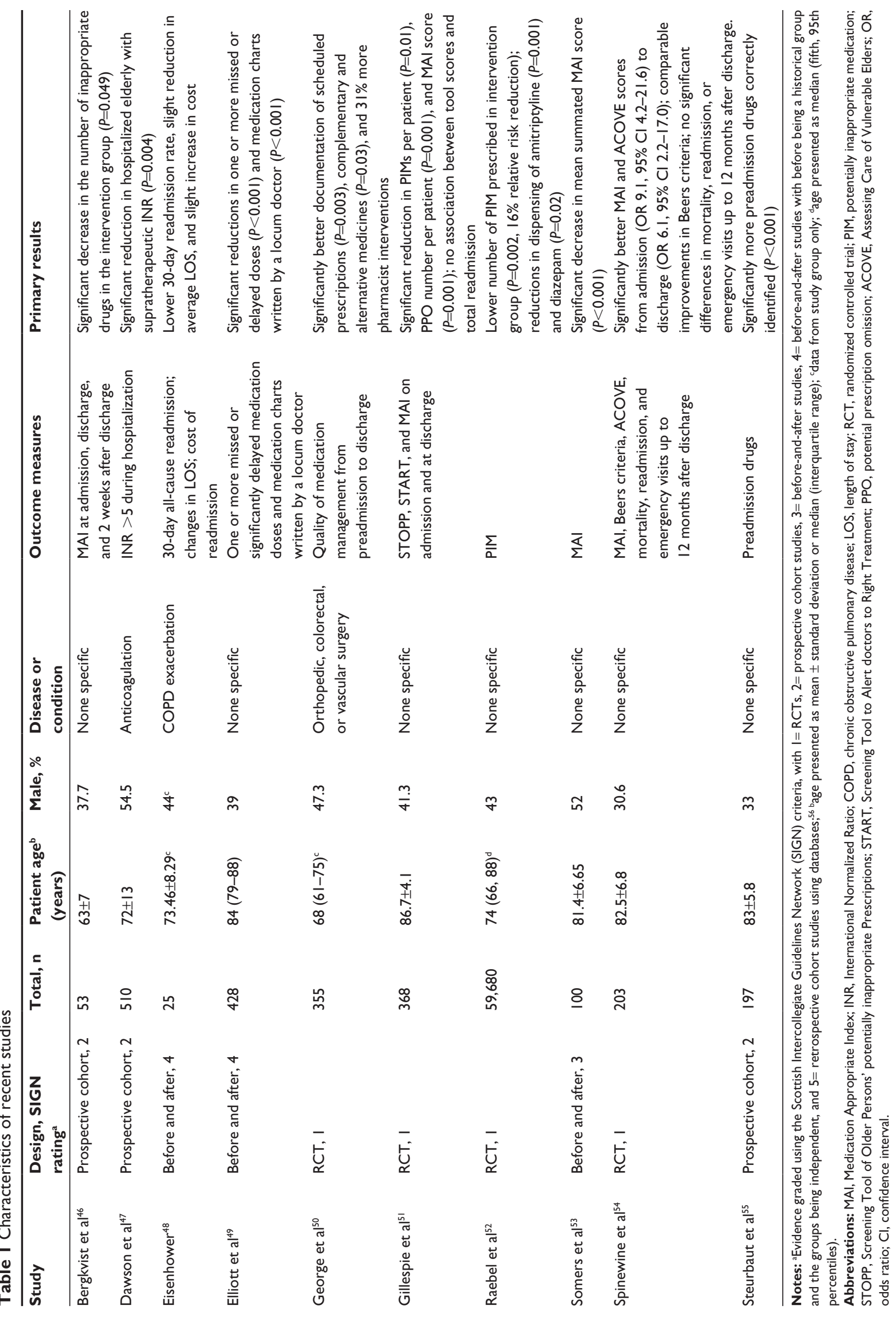




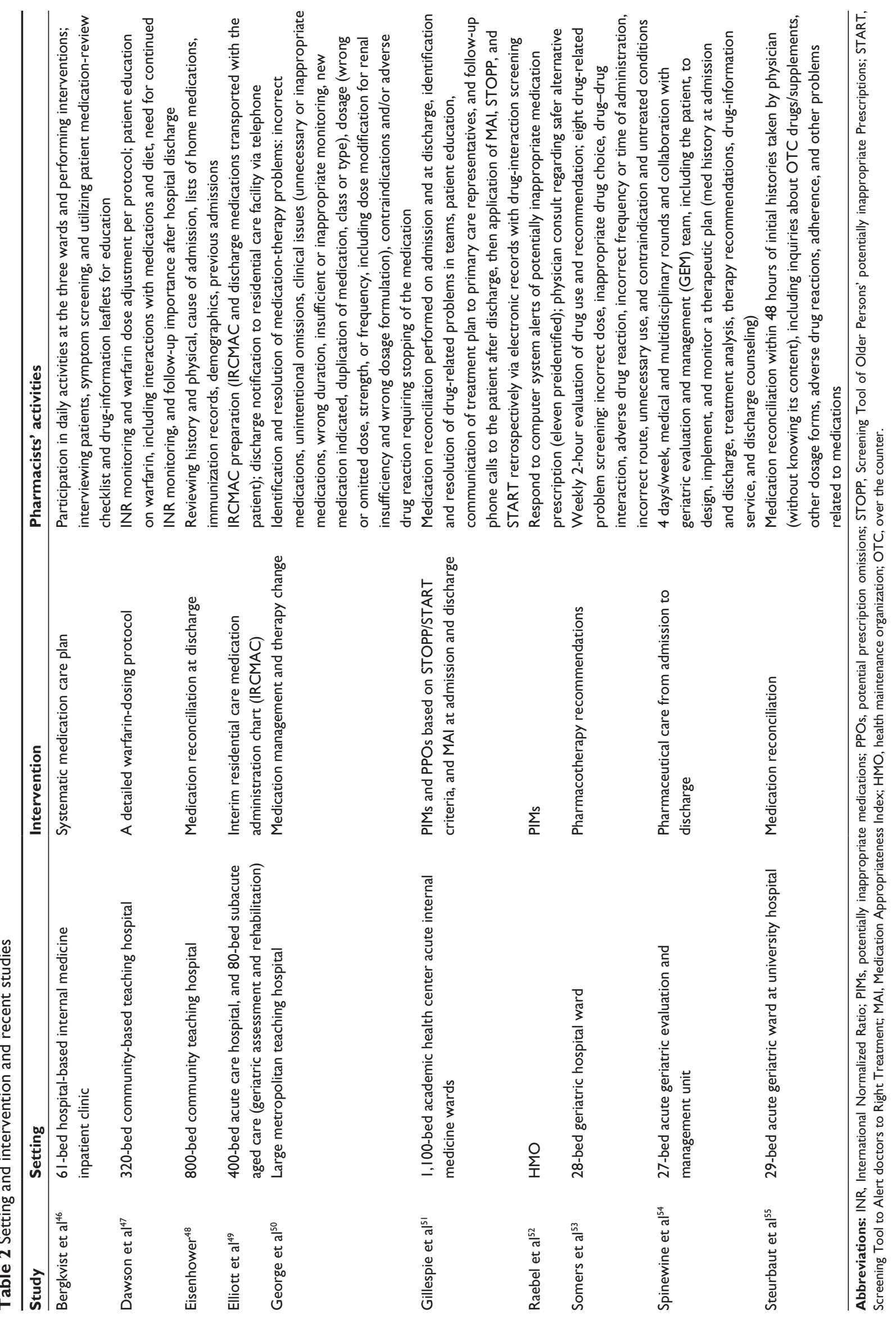


pharmacist-provided services on older patients, reporting significant benefit from pharmacist care in the intervention group compared to the comparison group. One beforeand-after study that measured cost found a lowered 30-day readmission rate and decreased average length of stay, with slightly increased cost of care. ${ }^{48}$ Therefore, current evidence of positive geriatric patient-oriented outcome achievement by pharmacists' interventions supports the previously published findings. Also, the impact can be translated internationally across aging countries.

\section{Role of pharmacists in geriatric care Polypharmacy and adherence management}

A 2003 systematic review examined the role of the pharmacist in polypharmacy reduction of older patients, which identified 14 studies at the time with varied aims and outcome measures. ${ }^{57}$ The studies showed clearly favorable results of pharmacists' services on polypharmacy reduction and prescribing quality, but most were not designed to demonstrate the impact of these results on patients' health or quality of life of older adults. ${ }^{57}$ More recently, enhanced research with better study design has clearly validated pharmacists' services of polypharmacy reduction and improved medication management leading to positive patient-oriented health outcomes..$^{21,46,51}$ Therefore, there is strong evidence for the role of pharmacists in providing geriatric care to reduce polypharmacy and clinical consequences of polypharmacy, including medication errors, nonadherence, adverse drug events, drug-drug interactions, urgent or emergent visits, and hospitalization.

It is evident that if patients do not use the medications prescribed to them, the benefits of those medications are negated completely. With intimate knowledge of medications and impact of medication nonadherence, pharmacists can simplify complex regimens and employ helpful tools to assist older adults adhere to their appropriate polypharmacy. ${ }^{21}$ Educational, technical, and behavioral approaches may be combined to correct patients' misunderstandings about their disease states or medications, and help ease the burden of having to be organized and to remember to take the medications at the right time to bring about positive behavioral changes. Such interventions can produce improvements in medication adherence that can extend to improved clinical outcomes for older patients on polypharmacy. $4,21,40,43$

As older adults acquire multiple chronic conditions, they accumulate a number of prescribing providers for those conditions. It has been shown that as the number of prescribers increased, the number of potentially harmful medications and adverse drug events reported by older adults increased. ${ }^{32,58}$ Older adults need a health care advocate and medication expert who can make sense of the treatment plans and prescriptions that are given by the multiple prescribers. Pharmacists can certainly play the role of this expert advocate, and yield safer and more effective medication regimens for the growing older adult populations. ${ }^{35,36,39,44,52}$

\section{Chronic disease management}

Older patients most often suffer from chronic diseases, including asymptomatic conditions, such as hypertension and dyslipidemia. Pharmacists having extensive knowledge of medications to treat chronic conditions and an up-to-date stance on clinical guidelines can provide impactful care to older populations. Favorable impacts of pharmacist care have been delineated by 30 studies included in a systematic review previously mentioned leading to reductions in blood pressure, total cholesterol, low-density lipoprotein cholesterol and smoking. ${ }^{11}$ Pharmacist care consisted of patient education, adherence interventions, cardiovascular risk assessment, medication management with communication to providers, or provider education. ${ }^{11}$ Among many conditions experienced by older adults, hypertension is the most commonly occurring chronic disease. Effective management of hypertension by pharmacists has been clearly demonstrated in the literature, with sensitive association of reductions in systolic blood pressure shown. ${ }^{14}$ Other examples include diabetes, ${ }^{15,16,35}$ heart failure, ${ }^{18} \mathrm{COPD},{ }^{19}$ anticoagulation, ${ }^{42,47}$ and osteoporosis..$^{40,59} \mathrm{Clearly}$, there is a role for pharmacists to provide chronic disease management to older adults within various health care systems and teams.

\section{Medication-error prevention during transition points}

Preventable medication errors are highly prevalent during transition points in older patients' care, whether they are moving from home to hospital, or hospital to home, rehabilitation facility, or nursing home. One study found that repeated medication errors were common (37.3\%) in the nursing home, which occurred more often in older ( $\geq 75$ years) and cognitively impaired residents, and were associated with greater harm than unrepeated errors. ${ }^{60}$ Most frequently repeated errors included wrong dosage and wrong administration. ${ }^{60}$ Medication errors around transition into a nursing home were assessed by a study conducted in the USA, with over 27,000 medication errors reported in the 3 -year period and $11 \%$ of the errors occurring during transition points. ${ }^{61}$ 
Through multivariate logistic regression, the study authors found that errors taking place during transition caused more harm to the patient than errors arising away from transition. ${ }^{61}$

In Northern Ireland, an integrated medicine-management service delivered by pharmacists at admission, inpatient stay, and discharge significantly lowered length of stay by 2 days over a 2 -month period $(P=0.027)$, and increased time to readmission by 20 days $(P=0.036) .{ }^{62}$ The integrated medicine-management service's data analysis indicated a number needed to treat of $12 .^{62}$ Kilcup et al assessed postdischarge medication reconciliation performed via phone by pharmacists within patient-centered medical homes and impact on patient readmission and cost savings. ${ }^{24}$ Significantly decreased readmission rates at 7 and 14 days and financial savings of US\$35,000 per 100 patients who received the intervention were realized. ${ }^{24}$ These positive findings from medication reconciliation by pharmacists around hospital transitions that led to decreased readmission rates and cost savings can be translated to older patient populations who are more vulnerable to transition-related medication misadventures. Several studies included in the current review have focused the pharmacists' effort on transition points and have found clinically significant benefits, solidifying their role. ${ }^{46,48,50,51,54}$

\section{Interprofessional team care}

Interprofessional team-based care has often been posed as a solution to growing older populations with heavy chronic disease and medication burden. The roles of pharmacists have evolved from being a chemist to drug dispenser and basic educator to now direct patient care provider serving as medication expert within interprofessional teams in diverse settings. ${ }^{63}$ As emphasized earlier, pharmacists have distinct expertise that can contribute to team knowledge and competence in managing older patients with polypharmacy and multiple chronic conditions. ${ }^{4}$ A 2010 Cochrane review identified 43 studies where pharmacist services were targeted directly at patients $(n=36)$ or other health care professionals $(n=7) .{ }^{63}$ Most studies included in the review favored pharmacists providing medication and therapeutic management, patient education, and provider education to improve clinical outcomes and prescribing patterns. ${ }^{63}$ In team care settings, the acceptance rate of pharmacist recommendations is high, as one European study noted, where $80 \%$ of 263 valid, documented recommendations were accepted by physicians in a geriatric hospital. ${ }^{64}$ The recommendations made most often by pharmacists included dose adjustment, administration time and frequency, and medication discontinuation. ${ }^{64}$ Additionally, a systematic review looking at pharmacist care in directed or collaborative fashion to manage heart failure retrieved 12 RCTs that significantly lowered all-cause hospitalization and heart failure-related hospitalization, with collaborative care having greater effects in reducing heart failure-related hospitalization compared to directed care. ${ }^{18}$ As mentioned earlier, there is clear evidence for the distinct role of pharmacists and their impact within health care teams that care for older adults. ${ }^{4,65}$ Therefore, one of the pharmacist's roles should be to contribute valuable expertise to team-based care of older patients.

\section{Tools for pharmacotherapy optimization in elders}

Medication misadventure, such as medication errors, inappropriate prescribing, suboptimal prescribing, and adverse drug events, is still common among older patients and continues to occur globally. ${ }^{66}$ Multiple validated tools are available for use to ensure appropriate medication use in older adults. Examples include the Beers criteria, ${ }^{27}$ STOPP (Screening Tool of Older Persons' potentially inappropriate Prescriptions) criteria, ${ }^{67}$ START (Screening Tool to Alert doctors to Right Treatment) criteria, ${ }^{67}$ and the MAI. ${ }^{68,69}$

Many medications are included in the tools for tracking inappropriate medications for older adults. For instance, the 2012 Beers criteria contain 53 medications and medication classes that are considered high risk in older adults, including benzodiazepine, sedative hypnotics, psychotropic agents, and anticholinergics. ${ }^{27}$ The STOPP criteria have been developed and are used commonly in Europe, and contain similar medications as the Beers criteria, with more specific details. ${ }^{67}$ Patterson et al conducted a Cochrane review to determine the effectiveness of the aforementioned tools in improving appropriate polypharmacy use in older adults, and found that studies using such tools led to reductions in inappropriate use of polypharmacy and inappropriate prescribing. ${ }^{70} \mathrm{~A}$ recently published Italian study reported that using a combination of Beers criteria and STOPP criteria to assess medications of hospitalized older adults led to a higher number of potentially inappropriate drugs identified than either alone. ${ }^{71}$ With rampant ageism in modern societies, underuse of potentially beneficial medications is also a problem for older adults, as one study reported occurrence of undertreatment in $62 \%$ of the participants. ${ }^{72}$ Most frequently omitted medications in the study included nitrates for patients post-myocardial infarction, multivitamins for the malnourished, and inhaled anticholinergic agents for patients with COPD. ${ }^{72}$ 
One of the included RCTs in the current review sought to measure the effects of pharmacists' interventions using the MAI, STOPP, and START on prescribing appropriateness for hospitalized older adults and care utilization in a 12-month follow-up period. ${ }^{51}$ Significant reductions in PIMs, potential prescription omissions, and MAI score were seen, with positive association detected between MAI and STOPP scores and drug-related readmissions. ${ }^{51}$ Therefore, it was shown that pharmacists' interventions using such tools have an affirmative impact on older patients and health care systems.

Because many medications causing anticholinergic symptoms are culprits of causing adverse drug events resulting in harmful and costly consequences among older people, multiple scales have been developed to gauge anticholinergic burden in older adults' medication regimens. A systematic review by Durán et al identified seven risk scales that delineated 47 high-potency anticholinergic drugs and 53 lowpotency anticholinergic drugs. ${ }^{73}$ Another recent systematic review by Salahudeen et al aimed to compare anticholinergic risk scales and association with adverse outcomes among older adults. ${ }^{33}$ They also identified seven anticholinergic risk scales, but found inconsistencies among the scales in terms of rating the medications' anticholinergic activity. This review concluded that the Anticholinergic Cognitive Burden scale ${ }^{74}$ was validated most frequently for adverse outcomes, as per their citation analysis. ${ }^{33}$ In a separate study, Salahudeen et al compared nine published anticholinergic risk scales and their association with negative outcomes in older adults. ${ }^{75}$ Even though the prevalence of anticholinergic exposure differed widely among the scales, all nine scales were significantly associated with hospitalization, admissions for falls, length of hospital stay, and general practitioner visits. The Drug Burden Index's anticholinergic component scores, ${ }^{76}$ age 85 years or greater, female sex, and polypharmacy use were the strongest predictors of the adverse outcomes. ${ }^{75}$

\section{Need for future research}

There is a paucity of data demonstrating clinically significant improvements when providing interventions to improve appropriate polypharmacy in older adults. ${ }^{70}$ In 2014, a systematic review aimed to determine the impact of discontinuing anticholinergic agents on cognitive function in older adults. ${ }^{77}$ Among the studies identified, only two cohort studies found improved cognitive performance after anticholinergic discontinuation. The authors concluded that further studies with larger sample size and longer follow-up are needed to better quantify the effects of discontinuing anticholinergic agents on cognitive function. ${ }^{77}$ In addition, a Cochrane review looking at effects of interventions to optimize medication prescribing targeting care-home residents found that the interventions effectively resolved medication-related problems, but there were no studies measuring changes in quality of life of the residents..$^{25}$ The authors concluded that studies evaluating clinical interventions to optimize medication appropriateness in care-home populations needed also to assess resident-related health outcomes. ${ }^{25}$ Therefore, future studies need to make greater efforts to tie clinical outcome measures with appropriate polypharmacy use among older patients.

When a systematic review conducted in Canada looked at the payment systems for pharmacist care in Canada, the USA, Europe, Australia, and New Zealand, they found highly variable pay systems for services provided and few programs tracking service outcomes. ${ }^{10}$ The authors urged future studies to analyze time required to perform pharmacists' interventions and outcomes attained to ensure adequate fees for program sustainability. ${ }^{10}$ Additionally, cost-effectiveness studies on geriatric pharmacy care models can enhance the provision of these services in broader settings globally and would delineate the cost of interventions that can derive clinical benefits in older patients, which may help the planning and implementation of such care.

Furthermore, the terminology used in the studies demonstrating pharmacist-delivered interventions is widely varied. One can find sundry descriptions used to categorize interventions provided by pharmacists. For example, one of the included studies in the current review called pharmacists' interventions to improve medication use and medication prescribing in older adults "medication management", 50 whereas another study named it "systematic medication care plan", ${ }^{46}$ and yet another study "pharmaceutical care". ${ }^{54}$ The measured clinical outcomes also vary among studies evaluating pharmacists' impact. For instance, some studies target lowering 3-day readmission rate, other studies 7-day readmission rate, and yet others 30 -day readmission rate. To some degree, the utility of defined outcome measures would depend on the types of interventions provided and for which condition, but any degree of standardization can add to the potency of impact demonstrated. Therefore, certain standardization of languages used for pharmacists' interventions and outcomes measured may be explored in future studies examining geriatric care by pharmacists.

\section{Conclusion}

Across various practice settings and diseases managed, pharmacists are actively engaged in improving pharmacotherapy 
for older patients. The current review strongly supports previous findings that showed positive impact of pharmacists' interventions on older patients' health-related outcomes. Therefore, there is a clear role for pharmacists' direct or collaborative care to optimize pharmacotherapy in older adults, and global health care teams caring for elders should involve pharmacists.

\section{Disclosure}

The authors report no conflicts of interest in this work.

\section{References}

1. Isasi F, Krofah E. The Expanding Role of Pharmacists in a Transformed Health Care System. Washington: National Governors Association Center for Best Practices; 2015.

2. Lewis NJ, Shimp LA, Rockafellow S, Tingen JM, Choe HM, Marcelino MA. The role of the pharmacist in patient-centered medical home practices: current perspectives. Integr Pharm Res Pract. 2014;3: 29-38.

3. Chisholm-Burns MA, Kim Lee J, Spivey CA, et al. US pharmacists' effect as team members on patient care: systematic review and metaanalyses. Med Care. 2010;48(10):923-933.

4. Lee JK, Slack MK, Martin J, Ehrman C, Chisholm-Burns M. Geriatric patient care by U S pharmacists in healthcare teams: systematic review and meta-analyses. J Am Geriatr Soc. 2013;61(7):1119-1127.

5. Fleming A, Browne J, Byrne S. The effect of interventions to reduce potentially inappropriate antibiotic prescribing in long-term care facilities: a systematic review of randomised controlled trials. Drugs Aging. 2013;30(6):401-408.

6. Tan EC, Stewart K, Elliott RA, George J. Pharmacist consultations in general practice clinics: the Pharmacists in Practice Study (PIPS). Res Social Adm Pharm. 2014;10(4):623-632.

7. Deschodt M, Flamaing J, Haentjens P, Boonen S, Milisen K. Impact of geriatric consultation teams on clinical outcome in acute hospitals: a systematic review and meta-analysis. $B M C \mathrm{Med}$. 2013;11:48.

8. Thomas R, Huntley AL, Mann M, et al. Pharmacist-led interventions to reduce unplanned admissions for older people: a systematic review and meta-analysis of randomised controlled trials. Age Ageing 2014;43(2): 174-187.

9. Pande S, Hiller JE, Nkansah N, Bero L. The effect of pharmacistprovided non-dispensing services on patient outcomes, health service utilisation and costs in low- and middle-income countries. Cochrane Database Syst Rev. 2013;2:CD010398.

10. Houle SK, Grindrod KA, Chatterley T, Tsuyuki RT. Paying pharmacists for patient care: a systematic review of remunerated pharmacy clinical care services. Can Pharm J (Ott). 2014;147(4):209-232.

11. Santschi V, Chiolero A, Burnand B, Colosimo AL, Paradis G. Impact of pharmacist care in the management of cardiovascular disease risk factors: a systematic review and meta-analysis of randomized trials. Arch Intern Med. 2011;171(16):1441-1453.

12. Santschi V, Chiolero A, Colosimo AL, et al. Improving blood pressure control through pharmacist interventions: a meta-analysis of randomized controlled trials. J Am Heart Assoc. 2014;3(2):e000718.

13. Machado M, Bajcar J, Guzzo GC, Einarson TR. Sensitivity of patient outcomes to pharmacist interventions. Part II: Systematic review and meta-analysis in hypertension management. Ann Pharmacother. 2007;41(11):1770-1781.

14. Machado M, Nassor N, Bajcar JM, Guzzo GC, Einarson TR. Sensitivity of patient outcomes to pharmacist interventions. Part III: Systematic review and meta-analysis in hyperlipidemia management. Ann Pharmacother. 2008;42(9):1195-1207.
15. Machado M, Bajcar J, Guzzo GC, Einarson TR. Sensitivity of patient outcomes to pharmacist interventions. Part I: Systematic review and meta-analysis in diabetes management. Ann Pharmacother. 2007; 41(10):1569-1582.

16. Wubben DP, Vivian EM. Effects of pharmacist outpatient interventions on adults with diabetes mellitus: a systematic review. Pharmacotherapy. 2008;28(4):421-436.

17. Jameson JP, Baty PJ. Pharmacist collaborative management of poorly controlled diabetes mellitus: a randomized controlled trial. Am J Manag Care. 2010;16(4):250-255.

18. Koshman SL, Charrois TL, Simpson SH, McAlister FA, Tsuyuki RT. Pharmacist care of patients with heart failure: a systematic review of randomized trials. Arch Intern Med. 2008;168(7):687-694.

19. Zhong H, Ni XJ, Cui M, Liu XY. Evaluation of pharmacist care for patients with chronic obstructive pulmonary disease: a systematic review and meta-analysis. Int J Clin Pharm. 2014;36(6):1230-1240.

20. Kim J, Tofade TS, Peckman H. Caring for the elderly in an inpatient setting: managing insomnia and polypharmacy. $J$ Pharm Pract. 2009;22(5):494-506.

21. Lee JK, Grace KA, Taylor AJ. Effect of a pharmacy care program on medication adherence and persistence, blood pressure, and low-density lipoprotein cholesterol: a randomized controlled trial. JAMA. 2006; 296(21):2563-2571.

22. Tan EC, Stewart K, Elliott RA, George J. Pharmacist services provided in general practice clinics: a systematic review and meta-analysis. Res Social Adm Pharm. 2014;10(4):608-622.

23. Sen S, Bowen JF, Ganetsky VS, et al. Pharmacists implementing transitions of care in inpatient, ambulatory and community practice settings. Pharm Pract (Granada). 2014;12(2):439.

24. Kilcup M, Schultz D, Carlson J, Wilson B. Postdischarge pharmacist medication reconciliation: impact on readmission rates and financial savings. J Am Pharm Assoc. 2013;53(1):78-84.

25. Alldred DP, Raynor DK, Hughes C, Barber N, Chen TF, Spoor P. Interventions to optimise prescribing for older people in care homes. Cochrane Database Syst Rev. 2013;2:CD009095.

26. Lu WH, Wen YW, Chen LK, Hsiao FY. Effect of polypharmacy, potentially inappropriate medications and anticholinergic burden on clinical outcomes: a retrospective cohort study. CMAJ. 2015;187(4):E130-E137.

27. American Geriatrics Society 2012 Beers Criteria Update Expert Panel. American Geriatrics Society updated Beers Criteria for potentially inappropriate medication use in older adults. JAm Geriatr Soc. 2012;60(4): 616-631.

28. Linton A, Garber M, Fagan NK, Peterson MR. Examination of multiple medication use among TRICARE beneficiaries aged 65 years and older. J Manag Care Pharm. 2007;13(2):155-162.

29. Tulner LR, Frankfort SV, Gijsen GJ, van Campen JP, Koks CH, Beijnen JH. Drug-drug interactions in a geriatric outpatient cohort: prevalence and relevance. Drugs Aging. 2008;25(4):343-355.

30. Obreli Neto PR, Nobili A, de Lyra DP Jr, et al. Incidence and predictors of adverse drug reactions caused by drug-drug interactions in elderly outpatients: a prospective cohort study. J Pharm Pharm Sci. 2012;15(2): 332-343.

31. Slabaugh SL, Maio V, Templin M, Abouzaid SA. Prevalence and risk of polypharmacy among the elderly in an outpatient setting: a retrospective cohort study in the Emilia-Romagna region, Italy. Drugs Aging. 2010;27(12):1019-1028.

32. Nyborg G, Straand J, Brekke M. Inappropriate prescribing for the elderlya modern epidemic? Eur J Clin Pharmacol. 2012;68(7):1085-1094.

33. Salahudeen MS, Duffull SB, Nishtala PS. Anticholinergic burden quantified by anticholinergic risk scales and adverse outcomes in older people: a systematic review. BMC Geriatr. 2015;15:31.

34. Ponniah A, Anderson B, Shakib S, Doecke CJ, Angley M. Pharmacists' role in the post-discharge management of patients with heart failure: a literature review. J Clin Pharm Ther. 2007;32(4):343-352.

35. Aspinall SL, Zhao X, Good CB, et al. Intervention to decrease glyburide use in elderly patients with renal insufficiency. Am J Geriatr Pharmacother. 2011;9(1):58-68. 
36. Badger N, Mullis S, Butler K, Tucker D. Pharmacist's intervention for older hospitalized patients. Am J Health Syst Pharm. 2007;64(17): 1794-1796.

37. Blalock SJ, Casteel C, Roth MT, Ferreri S, Demby KB, Shankar V. Impact of enhanced pharmacologic care on the prevention of falls: a randomized controlled trial. Am J Geriatr Pharmacother. 2010;8(5):428-440.

38. Dedhia P, Kravet S, Bulger J, et al. A quality improvement intervention to facilitate the transition of older adults from three hospitals back to their homes. J Am Geriatr Soc. 2009;57(9):1540-1546.

39. Delate T, Chester EA, Stubbings TW, Barnes CA. Clinical outcomes of a home-based medication reconciliation program after discharge from a skilled nursing facility. Pharmacotherapy. 2008;28(4):444-452.

40. Heilmann RM, Friesleben CR, Billups SJ. Impact of a pharmacistdirected intervention in postmenopausal women after fracture. $\mathrm{Am} J$ Health Syst Pharm. 2012;69(6):504-509.

41. Koehler BE, Richter KM, Youngblood L, et al. Reduction of 30-day postdischarge hospital readmission or emergency department (ED) visit rates in high-risk elderly medical patients through delivery of a targeted care bundle. J Hosp Med. 2009;4(4):211-218.

42. Poon IO, Lal L, Brown EN, Braun UK. The impact of pharmacistmanaged oral anticoagulation therapy in older veterans. $J$ Clin Pharm Ther. 2007;32(1):21-29.

43. Schneider PJ, Murphy JE, Pedersen CA. Impact of medication packaging on adherence and treatment outcomes in older ambulatory patients. J Am Pharm Assoc (2003). 2008;48(1):58-63.

44. Smith SR, Catellier DJ, Conlisk EA, Upchurch GA. Effect on health outcomes of a community-based medication therapy management program for seniors with limited incomes. Am J Health Syst Pharm. 2006;63(4):372-379.

45. Suhrie EM, Hanlon JT, Jaffe EJ, Sevick MA, Ruby CM, Aspinall SL. Impact of a geriatric nursing home palliative care service on unnecessary medication prescribing. Am J Geriatr Pharmacother. 2009;7(1):20-25.

46. Bergkvist A, Midlöv P, Höglund P, Larsson L, Eriksson T. A multiintervention approach on drug therapy can lead to a more appropriate drug use in the elderly. LIMM-Landskrona Integrated Medicines Management. J Eval Clin Pract. 2009;15(4):660-667.

47. Dawson NL, Porter IE 2nd, Klipa D, et al. Inpatient warfarin management: pharmacist management using a detailed dosing protocol. J Thromb Thrombolysis. 2012;33(2):178-184.

48. Eisenhower C. Impact of pharmacist-conducted medication reconciliation at discharge on readmissions of elderly patients with COPD. Ann Pharmacother. 2014;48(2):203-208.

49. Elliott RA, Tran T, Taylor SE, et al. Impact of a pharmacist-prepared interim residential care medication administration chart on gaps in continuity of medication management after discharge from hospital to residential care: a prospective pre- and post-intervention study (MedGap study). BMJ Open. 2012;2(3):e000918.

50. George LJ, Senturk-Raif R, Hodgkinson MR, Emmerton M, Larmour I. Impact of a surgical preadmission clinic pharmacist on the quality of medication management from preadmission to discharge: a randomised controlled study. J Pharm Pract Res. 2011;41(3):212-216.

51. Gillespie U, Alassaad A, Hammarlund-Udenaes M, et al. Effects of pharmacists' interventions on appropriateness of prescribing and evaluation of the instruments' (MAI, STOPP and STARTs') ability to predict hospitalization - analyses from a randomized controlled trial. PLoS One. 2013;8(5):e62401.

52. Raebel MA, Charles J, Dugan J, et al. Randomized trial to improve prescribing safety in ambulatory elderly patients. $J$ Am Geriatr Soc. 2007;55(7):977-985.

53. Somers A, Robays H, De Paepe P, Van Maele G, Perehudoff K, Petrovic M. Evaluation of clinical pharmacist recommendations in the geriatric ward of a Belgian university hospital. Clin Interv Aging. 2013;8:703-709.

54. Spinewine A, Swine C, Dhillon S, et al. Effect of a collaborative approach on the quality of prescribing for geriatric inpatients: a randomized, controlled trial. J Am Geriatr Soc. 2007;55(5):658-665.

55. Steurbaut S, Leemans L, Leysen T, et al. Medication history reconciliation by clinical pharmacists in elderly inpatients admitted from home or a nursing home. Ann Pharmacother. 2010;44(10):1596-1603.
56. Scottish Intercollegiate Guidelines Network (SIGN). SIGN 50: A Guideline Developers' Handbook. Edinburgh: SIGN; 2014. Available from: http://www.sign.ac.uk/pdf/sign50.pdf. Accessed July 16, 2015.

57. Rollason V, Vogt N. Reduction of polypharmacy in the elderly: a systematic review of the role of the pharmacist. Drugs Aging. 2003;20(11): $817-832$.

58. Green JL, Hawley JN, Rask KJ. Is the number of prescribing physicians an independent risk factor for adverse drug events in an elderly outpatient population? Am J Geriatr Pharmacother. 2007;5(1):31-39.

59. Nadrash TA, Plushner SL, Delate T. Clinical pharmacists' role in improving osteoporosis treatment rates among elderly patients with untreated atraumatic fractures. Ann Pharmacother. 2008;42(3):334-340.

60. Crespin DJ, Modi AV, Wei D, et al. Repeat medication errors in nursing homes: contributing factors and their association with patient harm. Am J Geriatr Pharmacother. 2010;8(3):258-270.

61. Desai R, Williams CE, Greene SB, Pierson S, Hansen RA. Medication errors during patient transitions into nursing homes: Characteristics and association with patient harm. Am J Geriatr Pharmacother. 2011;9(6): 413-422.

62. Scullin C, Scott MG, Hogg A, McElnay JC. An innovative approach to integrated medicines management. $J$ Eval Clin Pract. 2007;13(5): 781-788.

63. Nkansah N, Mostovetsky O, Yu C, et al. Effect of outpatient pharmacists' non-dispensing roles on patient outcomes and prescribing patterns. Cochrane Database Syst Rev. 2010;(7):CD000336.

64. Vella E, Azzopardi LM. Evaluation of pharmacist clinical recommendations in a geriatric hospital. J EuroMed Pharm. 2011;(1):2-5.

65. Scott I, Jayathissa S. Quality of drug prescribing in older patients: is there a problem and can we improve it? Intern Med J. 2010;40(1):7-18.

66. Hanlon JT, Semla TP, Schmader KE. Medication misadventures in older adults: literature from 2013. J Am Geriatr Soc. 2014;62(10): 1950-1953.

67. O'Mahony D, O'Sullivan D, Byrne S, O'Connor MN, Ryan C, Gallagher P. STOPP/START criteria for potentially inappropriate prescribing in older people: version 2. Age Ageing. 2015;44(2): 213-218.

68. Hanlon JT, Schmader KE, Samsa GP, et al. A method for assessing drug therapy appropriateness. J Clin Epidemiol. 1992;45(10):1045-1051.

69. Fitzgerald LS, Hanlon JT, Shelton PS, et al. Reliability of a modified medication appropriateness index in ambulatory older persons. Ann Pharmacother. 1997;31(5):543-548.

70. Patterson SM, Cadogan CA, Kerse N, et al. Interventions to improve the appropriate use of polypharmacy for older people. Cochrane Database Syst Rev. 2014;10:CD008165.

71. Tosato M, Landi F, Martone AM, et al. Potentially inappropriate drug use among hospitalised older adults: results from the CRIME study. Age Ageing. 2014;43(6):767-773.

72. Wright RM, Sloane R, Pieper CF, et al. Underuse of indicated medications among physically frail older US veterans at the time of hospital discharge: results of a cross-sectional analysis of data from the geriatric evaluation and management drug study. Am J Geriatr Pharmacother. 2009;7(5):271-280.

73. Durán CE, Azermai M, Vander Stichele RH. Systematic review of anticholinergic risk scales in older adults. Eur J Clin Pharmacol. 2013; 69(7):1485-1496.

74. Kolanowski A, Fick DM, Campbell J, Litaker M, Boustani M. A preliminary study of anticholinergic burden and relationship to a quality of life indicator, engagement in activities, in nursing home residents with dementia. JAm Med Dir Assoc. 2009;10(4):252-257.

75. Salahudeen MS, Hilmer SN, Nishtala PS. Comparison of anticholinergic risk scales and associations with adverse health outcomes in older people. J Am Geriatr Soc. 2015;63(1):85-90.

76. Kersten H, Wyller TB. Anticholinergic drug burden in older people's brain - how well is it measured? Basic Clin Pharmacol Toxicol. 2014;114(2):151-159.

77. Salahudeen MS, Duffull SB, Nishtala PS. Impact of anticholinergic discontinuation on cognitive outcomes in older people: a systematic review. Drugs Aging. 2014;31(3):185-192. 
Integrated Pharmacy Research and Practice

Dovepress

\section{Publish your work in this journal}

Integrated Pharmacy Research and Practice is an international, peer-reviewed, open access, online journal, publishing original research, reports, reviews and commentaries on all areas of academic and professional pharmacy practice. This journal aims to represent the academic output of pharmacists and pharmacy practice with particular focus on integrated care. All papers are carefully peer reviewed

Submit your manuscript here: http://www.dovepress.com/integrated-pharmacy-research-and-practice-journal to ensure the highest standards as well as ensuring that we are informing and stimulating pharmaceutical professionals. The manuscript management system is completely online and includes a very quick and fair peer-review system, which is all easy to use. Visit http://www.dovepress.com/testimonials.php to read real quotes from published authors. 